\title{
Generation of Submicron Metal Particles
}

\author{
P. M. SHERMAN
}

Department of Aerospace Engineering, The University of Michigan, Ann Arbor, Michigan 48105

Received August 5, 1974; accepted November 14, 1974

\begin{abstract}
A low inductance circuit was designed, built and employed for the evaporation of metal wires with subsequent condensation of the metal vapor into small particles. The metal vapor was produced by the fast transfer of energy stored in a capacitor. A single pulse discharge with current and voltage going to zero simultaneously without the usual oscillation or dwell and restrike, was employed for each measurement of actual energy transferred to the wire (rather than energy stored). Silver, cadmium, and zinc wire were used in ambient atmospheres of air, of helium or of argon. The particles generated were measured by actual count employing enlarged photographs taken from electron microscope pictures of shadowed samples.

The particles generated were spherical in shape and in the submicron range $(50-1,000 \AA)$, with roughly a $\log$ normal distribution of diameters. The mean diameter decreased with an increase in the characteristic length for the expansion based on the energy transferred. The kind of ambient gas appears to have at most a minor effect on the particle size generated (when there is no chemical reaction).
\end{abstract}

\section{INTRODUCTION}

The generation of small particulates have been of interest for many purposes. The colloidal energy converter (1), a colloidal thrustor (2), and colloidal electrostatic rocket engines (3) all require particles with good conducting properties or metallic composition. Submicron silver particles were also used for inhalation studies (4). Submicron metal particles were used for the generation of metal vapor by reevaporation (5), (6), and were generated in the study of exploding wire phenomena (7). A new interest in particulates is a result of the laser-Doppler measurements of velocity which require seeding of the fluid medium (8).

One simple way of generating small metallic particles is by transfering a large amount of energy very fast to a thin wire "exploding" it. Vaporization of the wire and condensation of the metal vapor occurs in microseconds, and the particles generated are very small. There are many papers relating to exploding wire phenomena (9). However, a review of the liter- ature reveals only a direct measurement of particle size distribution and none which include both a direct measurement of the actual energy transferred to the wire and the particle size. Although the energy stored before discharge based on charge voltage and the capacitance employed $\left(\frac{1}{2} \mathrm{CV}^{2}\right)$ is generally noted, the energy actually transferred to the wire can be as little as $50 \%$ of the energy stored.

There is a summary (4) of measurements of the size of particles generated by exploding wires which indicates the disparity in the kinds of measurements made. Presented here are new data on the generation of small metallic particles employing electrical evaporation of thin wires, and condensation of the metal vapor generated. The new data include measurements of energy actually transferred to the wire in each case as well as of the particle size generated.

\section{EXPERIMENTAL ARRANGEMENT}

A simple way of obtaining heated metal vapor is accomplished by using a metal wire 


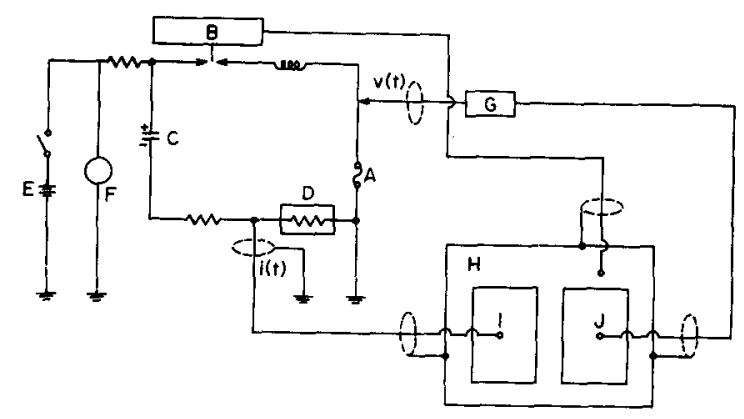

FIg. 1. Schematic of electrical circuit. (A) exploding wire; (B) spark gap trigger; (C) 14.7 microfarad condenser; (D) current viewing resistor $\left(4.99 \times 10^{-4} \Omega\right)$; (E) $30 \mathrm{kV}$ power supply; (F) voltmeter; (G) 1000:1 P-6015 high voltage probe; (H) Tektronix type 551 dual beam oscilloscope; (I) type L plug-in; (J) type CA plug-in.

as the load of a low inductance LRC circuit. A circuit was designed with low inductance $(<1 \mu \mathrm{H})$ for fast energy transfer. Figure 1 shows a schematic of the system used. The energy is stored in a low inductance condenser (14.7 $\mu \mathrm{f}$ Sangamo) which stores up to $3,000 \mathrm{~J}$ at $20,000 \mathrm{~V}$. A spark gap is employed to initiate the discharge. Current is measured as a function of time by means of a current viewing (noninductive) resistor ( $\mathrm{T}$ and $\mathrm{M}$ Company). Voltage across the wire is measured as a function of time with a P-6015 Tektronix High Voltage Probe. A single pulse discharge explored previously (10), in which current and voltage go to zero simultaneously without the usual dwell or restrike or oscillation, was employed. The voltage and current traces of such a discharge are shown in Fig. 2. Energy actually transferred to the wire as a function of time can then be determined from the current and voltage traces by integrating the product of current and voltage over the time period of the discharge. Figure 3 shows a graph of energy transfer as a function of time from such an integration of current times voltage.

The circuit was designed to take wires of different lengths and diameters in a chamber for variable ambient gases. The wire to be

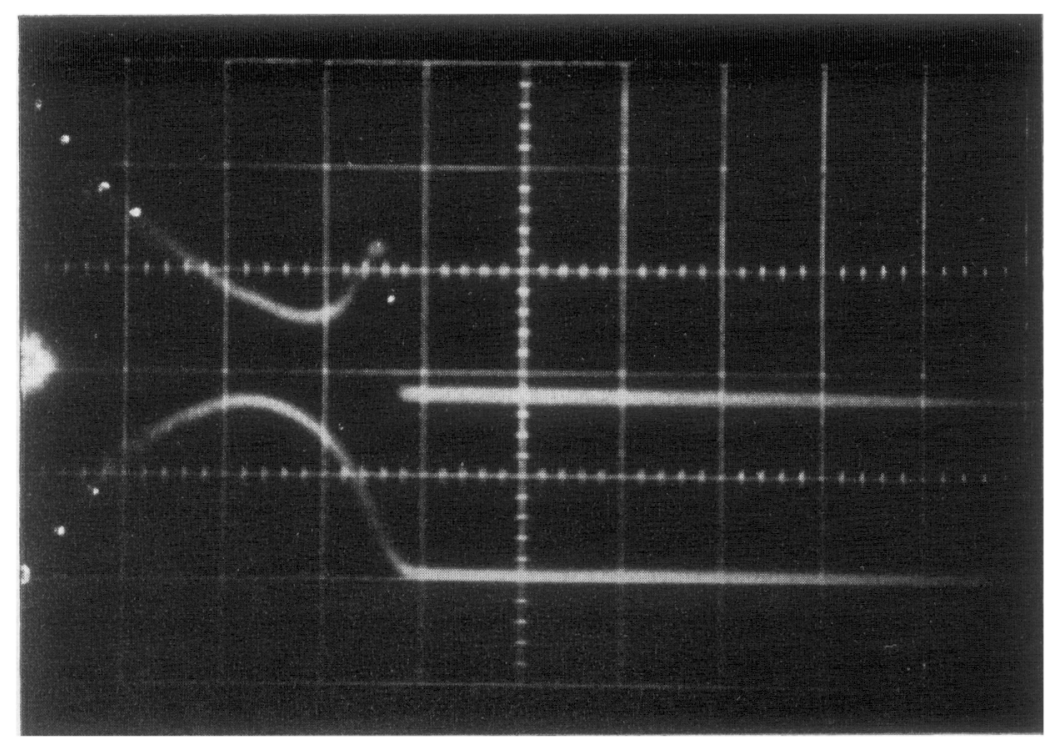

Fig. 2. Current and voltage traces. Upper trace, $2 \mathrm{kV} /$ div; lower trace, $20 \mathrm{kA} /$ div; sweep speed, 1 microsecond/div. 


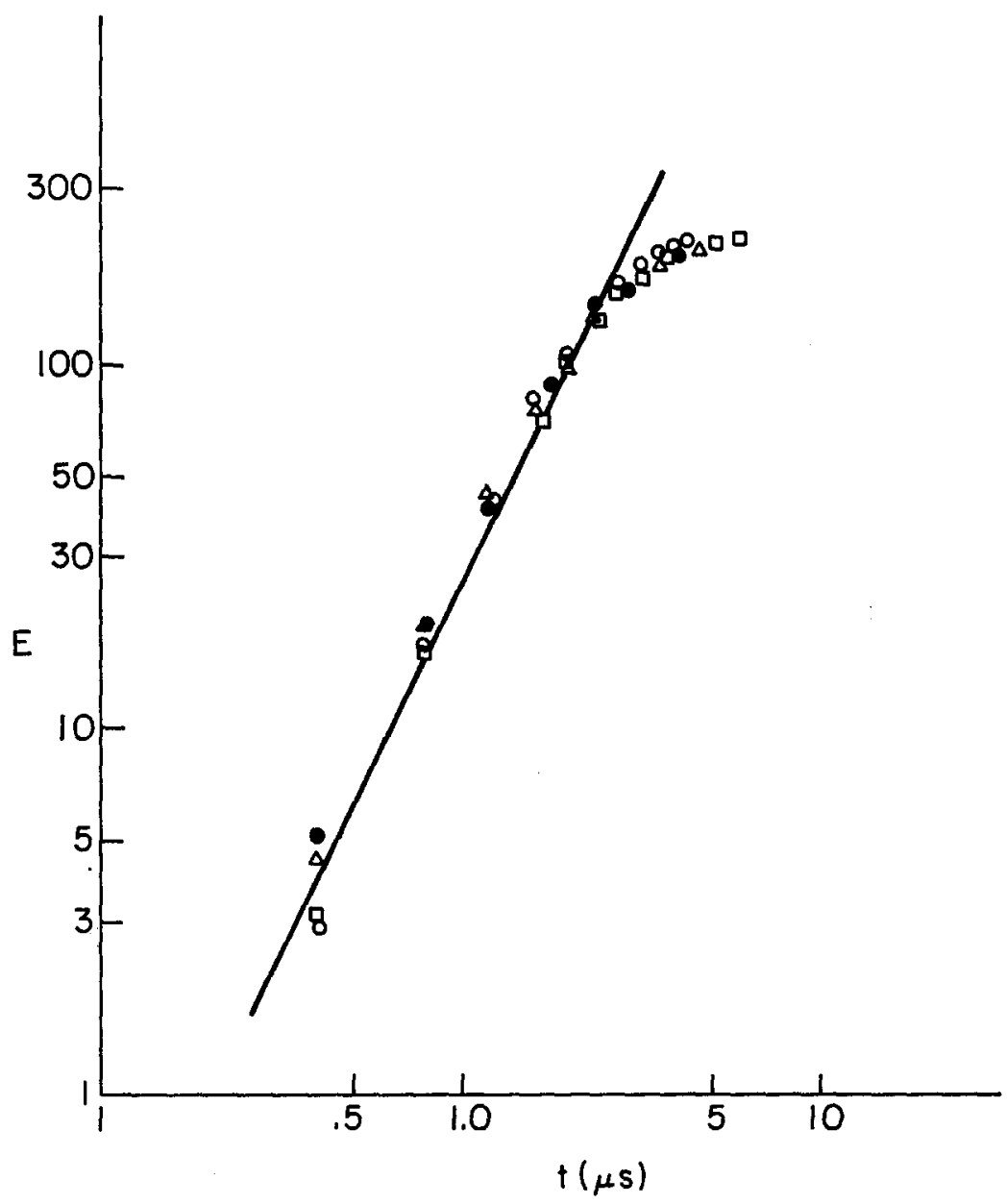

FIG. 3. Energy transferred in Joules as a function of time in microseconds.

evaporated was bounded on each end by a Plexiglas plate covering the electrodes so that a true cylindrical expansion of metal vapor takes place. Particles were collected on thin strips of coated mica pasted to the Plexiglas plate. Particles formed a dust cloud which settled reasonably fast on all surfaces as a result of their kinetic motion. There was no change in particle size distribution with settling time after a period of several minutes.

The particles collected were too small to be seen by an optical microscope so an electron microscope of the transmitted beam type was employed. The collecting surface was a carbon layer evaporated over mica strips cleaved along lattice surfaces for microscopic smoothness.
The collected particles were shadowed with chromium evaporated at an angle to permit three-dimensional analyses of the collected particles. The carbon layer was placed on the microscope copper grid by floating it off the mica surface in water.

An enlarged electron microscope photograph of particles is shown in Fig. 4. The white "tails" are shadows indicating the height of each particle above the collecting surface. As can be seen agglomeration was not a problem.

Silver wire was primarily used. It has the advantage of permitting the ambient gas to be air and still avoiding a chemical reaction. Silver was also used in helium and in argon as the ambient media. Zinc and cadmium 
wires were used in helium as the ambient gas. checked. Wire lengths between 3.8 and $5 \mathrm{~cm}$ The ambient gas was at atmospheric pressure were employed in No. 22 to No. 26 gauge wire. in all cases. The energy transferred to the wire Size adjustments were made to keep the energy was measured in each case. Energy necessary transferred greater than the energy necessary to evaporate the wire was calculated and for evaporation.

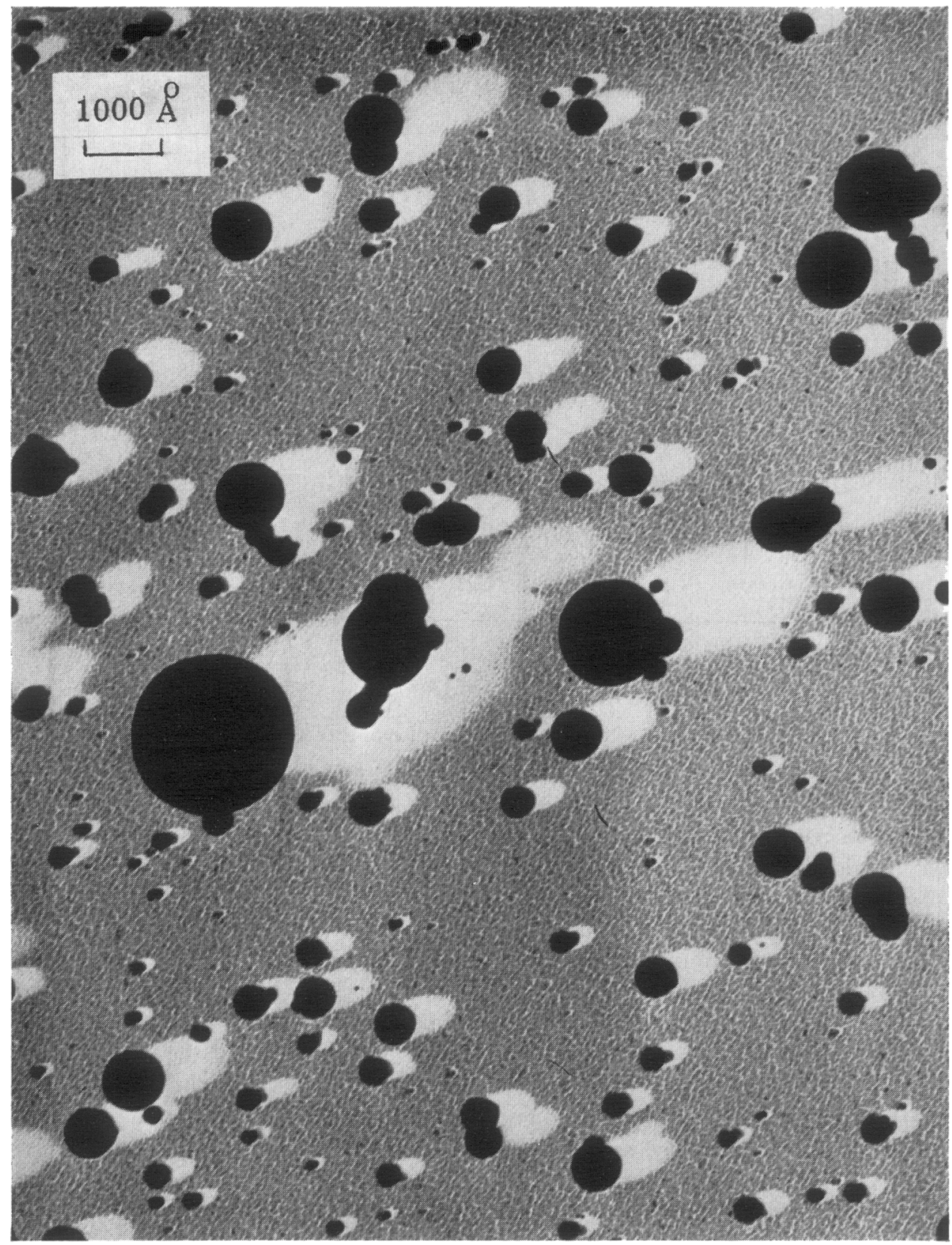

Fig. 4. Photomicrograph of chromium shadowed silver particles. 


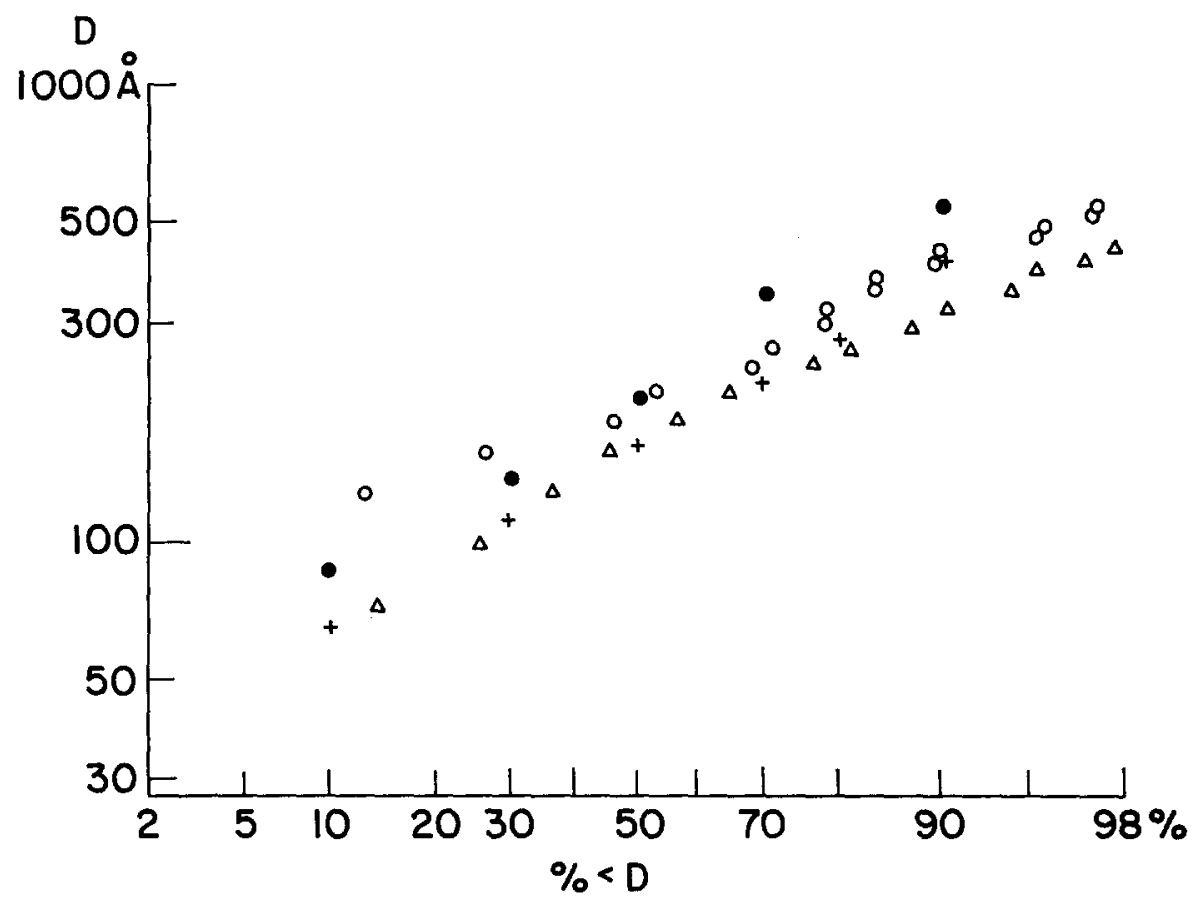

Fic. 5. Cumulative size distribution of silver particles. Triangles are wires in helium as the ambient gas; circles are in argon; pluses and dots are in air as the ambient gas.

\section{RESULTS AND DISCUSSION}

The particles generated by the evaporation of thin metal wires and the condensation of the metal vapor formed were all spherical in shape when no chemical reaction between metal vapor and ambient gas takes place. The shape of the particles can be seen in Fig. 4 which is an electron microscope photograph of the particles generated shadowed at an angle with chromium to show the three-dimensional image. (When zinc was evaporated in air, the particles generated were no longer spherical in shape but rather hexagonal cylinders, presumably of zinc oxide rather than zinc and a result of the chemical reaction rather than phase change.) The spherical shape for condensed metal particles is certainly consistent with previous photographs of particles condensed (11).

The particle size distributions obtained for silver in air, in helium and in argon are shown in Fig. 5. The particle diameter in Angstroms is plotted versus the percent of particles smaller than the diameter $D$. These cumulative dis- tributions shown in log probability coordinates indicate that the distribution is roughly $\log$ normal (12). The kind of ambient gas appears to have at most a minor effect (when no chemical reaction is involved) on particle size. The samples were taken 3 in. away from the wire for the evaporation of silver wire in helium and in the argon. The samples were taken $1 \frac{1}{2}$ and $2 \frac{1}{2}$ in. away from the wire when air was the ambient gas. Some shift to larger particles with distance close in could be seen but distributions from $2 \frac{1}{2}$ to 4 in. away from the wire were essentially identical for samples taken at any given set of conditions. This can be seen in Fig. 6 where measurements are shown for samples taken simultaneously at 3 and 4 in. away from the wire for the cadmium wire evaporated in helium. The zinc in helium size distributions were identical for samples taken at distances 2,3 , and 4 in. away from the wire. The conditions employed for the results shown in Figs. 5 and 6 are roughly for the same characteristic length, $R_{0}(13) . \quad R_{0} \equiv E_{0} /\left(2 \pi l p_{0}\right)^{\frac{1}{2}}$, 


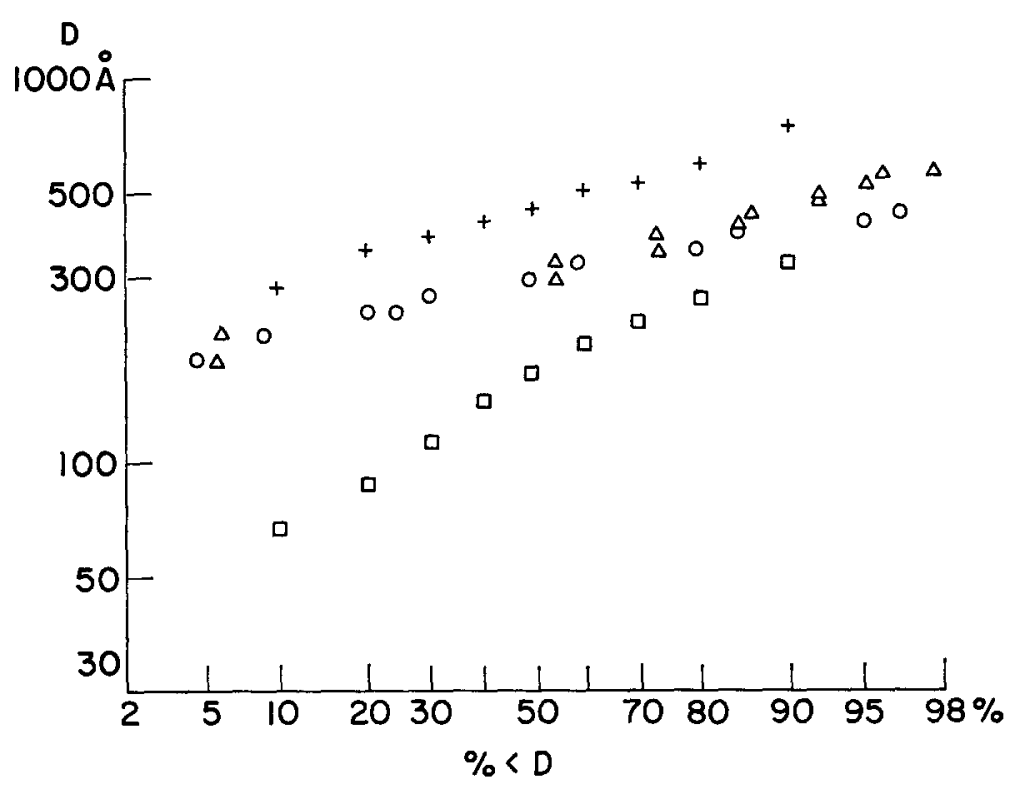

FIG. 6. Cumulative size distributions with helium as the ambient gas. Circles and triangles are for cadmium; pluses are for zinc; squares are for silver.

where $E_{0}$ is the energy transferred to the wire, $l$ is the length of the wire, and $p_{0}$ is the pressure of the ambient gas. $R_{0}$ can be considered the radius of a cylindrical volume of length $l$, which when swept out at the ambient gas pressure, $p_{0}$, would represent the energy transferred, $E_{0}$. $R_{0}$ is $8.5 \pm 0.4 \mathrm{~cm}$ for the results in Fig. 5 and $8.3 \pm 0.2 \mathrm{~cm}$ for the results in Fig. 6 .

Figure 6 appears to show that particles of zinc and of cadmium tend to have a narrower size distribution than that of silver (for similar characteristic lengths or relative energies transferred). This might be expected since silver has a larger surface tension and therefore a slower rate of nucleation yielding a wider spread of nuclei diameters. [The critical size particle for nucleation to proceed increases with surface tension and the number rate of formation of nuclei decreases exponentially with the increase in critical size particle squared, according to classical theory (14).]

The mean particle size is shown in Fig. 7 as a function of $R_{0}$, the characteristic length for the expansion based on the energy transferred. In general, the mean particle size decreases with increasing energy transferred. $R_{0}$ is a measure of the rate of expansion. The faster the expansion is, the greater the supersaturation and rate of nucleation. Hence the particles condensed would be expected to be smaller for the case of a faster expansion.

In conclusion it can be said that particles generated from condensation of "exploded" wires (when chemical reactions are not in-

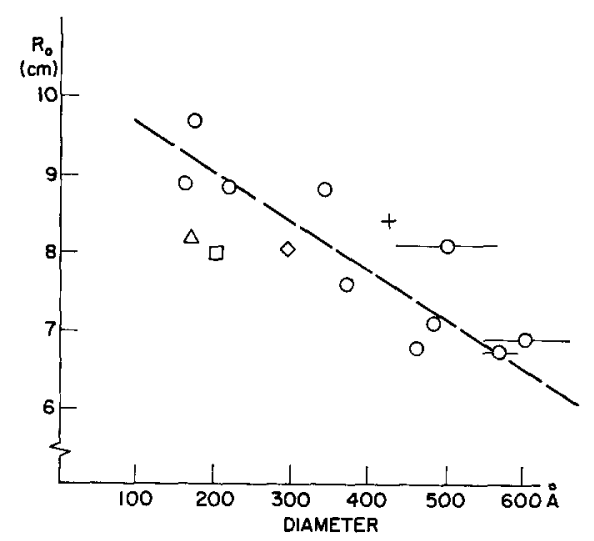

FIG. 7. Mean particle size as a function of characteristic length for the expansion, $R_{0}$, where $R_{0} \equiv$ $\left(E / 2 \pi l p_{0}\right)^{\frac{1}{2}}$. Circles are for silver in air; triangle is for silver in helium; square for silver in argon; plus for zinc in helium; diamond for cadmium in helium. 
volved) :

(a) are spherical in shape;

(b) can be represented roughly by a $\log$ normal size distribution;

(c) have a mean particle size roughly inversely proportional to the characteristic length for the expansion based on energy per unit length transferred to the wire;

(d) are at most marginally affected by the kind of ambient gas.

\section{ACKNOWLEDGMENTS}

This research was supported in part by the National Science Foundation under grant number GH 31855. The assistance of L. Griffin, G. Brooks, Z. Gabreijl, and D. Gell are gratefully acknowledged.

\section{REFERENCES}

1. Cox, A. L., Colloidal electrohydrodynamic energy convector, AIAA J. 1, 2491 (1963).

2. Goldin, D. S., A thermodynamic flow analysis of particle formation efficiency in a mixed flow colloid thruster, AIAA paper no. 67-85, fifth aerospace sciences meeting, 1967.

3. Norgren, C. T., "Onboard Colloidal Particle Generator for Electrostatic Engines," Progress in Astronautics and Aeronautics, Vol. 9. Academic Press, New York, 1963.
4. Phalen, R. F., Evaluation of an exploded-wire aerosol generator for use in inhalation studies, Aerosol Sci. 3, 395 (1972).

5. FALK, T. J., Research on hypersonic condensation phenomena in high temperature gases, U.S. Air Force rept. ARL 68-0143, Aug. 1968.

6. Sherman, P. M., McBride, D. D., and Oktay, E., Condensation in a rapidly expanding metal vaporinert gas mixtures, ARL report 67-0071, 1967.

7. Weber, F. N., AND ShEAR, D. D., Exploding wire particle size by light scattering measurement, J. Appl. Phys. 3854 (1969).

8. Penner, S. S., and Jerskey, T., "Annual Review of Fluid Mechanics," Vol. 5, p. 9, 1973.

9. Chace, W. G., and Moore, H. R., eds. "Exploding Wires," Vols. 1-4. Plenum Press, New York, 1959-1968.

10. Okтау, E., Effect of wire cross section on first pulse of an exploding wire, Rev. Sci. Inst. 36 (1965).

11. McBride, D. D., and Sherman, P. M., Condensed zinc particle size determined by a time discrete sampling apparatus, $A I A A J$. Aug. 1972.

12. SмtтH, J. E., AND Jordan, M. L., Mathematical and graphical interpretation of the $\log$ normal law for particle size distribution analysis, $J$. Colloid Sci. 19, 549 (1964).

13. SakURAI, A., Blast wave theory, in "Basic Developments in Fluid Dynamics," Vol. I. Academic Press, New York, 1965.

14. Frenkel, J., "Kinetic Theory of Liquids," Clarendon Press, Oxford, 1956. 